\title{
Magnetic Field Gradients and Their Uses in the Study of the Earth's Magnetic Field
}

\author{
C. G. A. HARrison and J. R. Southam \\ Rosenstiel School of Marine and Atmospheric Science, University of Miami, 4600 Rickenbacker Causeway, \\ Miami, Florida 33149, U.S.A.
}

(Received June 8, 1990; Revised February 27, 1991)

\begin{abstract}
Magnetic field gradient measurements for geophysical purposes have been made with much less frequency than total field observations. Yet there are considerable advantages in gradient measurements, especially if the objective is to study magnetic anomalies produced by magnetization contrasts in the crust and possibly upper mantle of the Earth. This is because gradient measurements enhance short wavelength signals relative to long wavelength signals. The power spectrum of a magnetic gradient measurement along any line is the same as the power spectrum of the field measured along the same line multiplied by the wavenumber. This means that signals from crustal sources are greatly enhanced over those from core sources, and also that diurnal variations of the field, which appear as very long wavelength features in most observations, are heavily attenuated compared with the crustal signal. To make full use of gradient measurements it is desirable to orient the gradient instrument. If orientation can be achieved, then it is possible to determine the lineation direction of lineated features using just one profile running at some angle to the feature lineation. This is similar to the technique for determining lineation direction from the measurement of field components, but the requirement for orientation is much less severe. Even if orientation cannot be achieved, there are rotationally invariant quantities which can be formulated from the gradient tensor which are of potential use in the interpretation of magnetic anomaly sources.
\end{abstract}

\section{Introduction}

One of the key papers in the interpretation of marine magnetic anomalies was that by BLAKELY et al. (1973) who took aeromagnetic field component data flown along a flight line which crossed the lineated magnetic anomalies in the NE Pacific just north of the Murray fracture zone (MASON, 1958; MASON and RAFF, 1961) approximately perpendicularly. This paper has not received the attention that it deserves, mainly because vector magnetic field data are difficult to gather. This is because the requirement for orientation is very severe. If we wish to make a component measurement accurate to 1 $\mathrm{nT}$ in the presence of a field of $50 \mu \mathrm{T}$, the orientation accuracy has to be $1 / 50000$ radians or $10^{-3}$ degrees. The actual accuracy of orientation achieved by BLAKELY et al. (1973) was much worse than this, some orientation errors being as large as 0.1 degrees.

BLAKELY et al. (1973) rotated the two horizontal components of field so that one component was parallel to the lineations and one component was perpendicular to the lineations. What they were able to show was that the anomalous component of field parallel to the lineations had a very small signal compared to the anomalous vertical and other horizontal component. A corollary of this observation is that it should be possible 
to determine if a set of anomalies is lineated, and the lineation direction, by observing the vector field along a straight line through the anomalies. In addition, if the anomalies are primarily lineated, it is possible to determine where nonlinearities exist by a simple transformation of the data. This consists of the following steps.

Suppose that the anomalous horizontal field in direction $\phi$ is $X_{1 \ldots n}$, and in direction $\phi+\pi / 2$ is $Y_{1 \ldots n}$, then it is possible to calculate the component in direction $\theta\left(Y_{1}^{\prime} \ldots n\right)$

$$
Y_{i}^{\prime}=X_{i} \cos (\theta-\phi)+Y_{i} \sin (\theta-\phi) .
$$

We wish to determine the angle $\theta$ which makes the anomalous variations of a component in this direction as small as possible, i.e. we wish to minimize

$$
\sum Y_{i}^{\prime 2}=\sum X_{i}^{2} \cos ^{2}(\theta-\phi)+\sum Y_{i}^{2} \sin ^{2}(\theta-\phi)+2 \sum X_{i} Y_{i} \sin (\theta-\phi) \cos (\theta-\phi)
$$

therefore,

$$
\frac{\partial}{\partial \theta} \sum_{i=1}^{n} Y_{i}^{\prime 2}=0
$$

This gives

$$
\tan 2(\theta-\phi)=\frac{2 \sum X_{i} Y_{i}}{\sum X_{i}^{2}-\sum Y_{i}^{2}}
$$

The two solutions for $\theta$ are separated by $\pi / 2$ and show the direction of the lineation and the perpendicular to the lineation.

The anomaly is then redefined with a vertical component $(z)$, a horizontal component perpendicular to the lineations $(x)$ and a horizontal component parallel to the lineations ( $y$ ) by using the appropriate value of $\theta$ from Eq. (4). The total field is generated from the vector addition of these components. Then from this total field anomaly, and assuming a knowledge of the direction of magnetization in the lineated sources, it is possible to generate $x$ and $z$ components assuming complete linearity by multiplication by hermitian functions in the wavenumber domain (BLAKELY and COX, 1972; BLAKELY et al., 1973). Comparison of these anomalies with the observed anomalies will reveal differences which if within a certain wavelength band are probably due to non-lineated sources. The same thing can be seen in the anomalies in the $y$ direction, which should be zero if complete linearity holds.

One problem already alluded to was that the orientation requirements for these vector field measurements are very severe, and it turned out that errors in orientation (about $0.1^{\circ}$ ) were responsible for short wavelength noise in the measurements. BLAKELY et al. (1973) were able to show that most of the signal at wavenumbers greater than 0.4 radians/ $\mathrm{km}$ (wavelengths shorter than $16 \mathrm{~km}$ ) were caused by this sort of noise.

ISEZAKI (1986) described a three component shipboard magnetometer for which the heading error is $\mathrm{l}^{\prime}$ and the vertical error is $5^{\prime}$. An error of $\mathrm{l}^{\prime}$ will give a component error as large as $15 \mathrm{nT}$ in the presence of a $50 \mu \mathrm{T}$ field. It was pointed out in this paper that component data can be especially useful in certain geometric situations. For instance, if 
lineations are running parallel to the horizontal field and the magnetization is in the same direction, then at the geomagnetic equator there will be no anomalous total field, whereas component data will give perfectly useful signals. Such a situation is closely approximated in the equatorial Atlantic. Further uses of this three component shipboard magnetometer are given by SEAMA and ISEZAKI (1990).

The possibility of using magnetic gradient measurements to study magnetic sources was first suggested by VESTINE and DAVIDS (1945). They showed that gradient measurements would be useful for looking at sources which are close to the observation point, and would be relatively uncontaminated by deep seated or long wavelength signals. As for the measurement of vector components of the field, if the magnetic gradient tensor is measured it is possible to determine the strike of lineation by a method similar to that outlined in Eqs. (1)-(4).

The magnetic gradient is a symmetric second rank tensor with zero trace and therefore has five independent quantities. There is often the possibility of measuring only one component of the tensor, usually the vertical gradient of the vertical field. Other quantities are also sometimes measured. For instance Hood $(1965,1975,1981)$, HoOD and MCCLURE (1965) and HOOD et al. (1979) used the vertical gradient of the total field in some applications. The relationship between this quantity and the gradient tensor is complicated, although at high latitudes, where the total field is almost vertical, it can approximate the vertical gradient of the vertical field.

\section{The Magnetic Gradient Tensor}

The magnetic gradient tensor is a second rank symmetric tensor with zero trace. It is shown below.

$$
\left.\mathbf{T}=\mid \begin{array}{lll}
\frac{\partial^{2} V}{\partial x^{2}} & \frac{\partial^{2} V}{\partial x \partial y} & \frac{\partial^{2} V}{\partial x \partial z} \\
\frac{\partial^{2} V}{\partial x \partial y} & \frac{\partial^{2} V}{\partial y^{2}} & \frac{\partial^{2} V}{\partial y \partial z} \\
\frac{\partial^{2} V}{\partial x \partial z} & \frac{\partial^{2} V}{\partial y \partial z} & \frac{\partial^{2} V}{\partial z^{2}}
\end{array}\right)
$$

Because the potential for a source free region satisfies Laplace's equation, the sum of the diagonals is equal to zero. Since the tensor is symmetric, there are only three independent off diagonal terms, and so the total number of independent terms is five.

Since we shall be dealing with spherical geometry, it will be convenient to describe the tensor in terms of the spherical coordinates used in spherical harmonic expansions of the Earth's magnetic field. In this case the gradient tensor becomes

$$
\begin{aligned}
& T_{r r}=\frac{\partial^{2} V}{\partial r^{2}}, \\
& T_{r \theta}=\frac{1}{r} \frac{\partial^{2} V}{\partial r \partial \theta}-\frac{1}{r^{2}} \frac{\partial V}{\partial \theta},
\end{aligned}
$$




$$
\begin{aligned}
& T_{r \phi}=\frac{1}{r \sin \theta} \frac{\partial^{2} V}{\partial \phi \partial r}-\frac{1}{r^{2} \sin \theta} \frac{\partial V}{\partial \phi}, \\
& T_{\theta \theta}=\frac{1}{r} \frac{\partial V}{\partial r}+\frac{1}{r^{2}} \frac{\partial^{2} V}{\partial \theta^{2}}, \\
& T_{\theta \phi}=\frac{1}{r^{2} \sin \theta} \frac{\partial^{2} V}{\partial \theta \partial \phi}+\frac{\cos \theta}{r^{2} \sin \theta} \frac{\partial V}{\partial \theta}, \\
& T_{\phi \phi}=\frac{1}{r} \frac{\partial V}{\partial r}+\frac{\cos \theta}{r^{2} \sin \theta} \frac{\partial V}{\partial \theta}+\frac{1}{r^{2} \sin \theta^{2}} \frac{\partial^{2} V}{\partial \phi^{2}} .
\end{aligned}
$$

This expression allows us to use spherical harmonic models of the Earth's magnetic field and calculate what all of the gradients are. Many of the expressions become quite complicated, but the expression for the vertical gradient of the vertical field (the first of the expressions above) is quite simple. For instance, the dipolar field of the Earth gives a vertical field of $61144 \cos \theta \mathrm{nT}$ where $\theta$ is the colatitude measured from the dipole axis. The vertical gradient of the vertical field is $28.79 \times 10^{-3} \cos \theta \mathrm{nT} / \mathrm{m}$. Other components of the gradient tensor are of comparable magnitude except for $T_{r \phi}$ which is everywhere zero.

The average power of the field in any degree of harmonic can be calculated using the Lowes-Mauersberger (MAUERSBERGER, 1956; LOWES, 1966) function.

$$
P(n)=(n+1) \sum_{m=0}^{n}\left(\left(g_{n}^{m}\right)^{2}+\left(h_{n}^{m}\right)^{2}\right)
$$

where $n$ is the degree and $m$ the order of the harmonic, and $g, h$, are the Gauss coefficients of the spherical harmonic expansion of the field. LOWES (1966) has also given mean square values of the vertical field over the sphere for each degree of harmonic, the multiplying factor before the summation sign being in this case $(n+1)^{2} /(2 n+1)$. The vertical gradient of the vertical field will have a mean square value which is obtained by multiplication by $[(n+1)(n+2) / r]^{2} /(2 n+1)$. A comparison of the power spectra of total field and $T_{r r}$ is given in Fig. 1.

Although the relative decrease in power of low degree harmonics compared with high degree harmonics appears to be quite modest in Fig. 1, this is not in fact true. We may define the power spectra for the gradient in a way similar to the result LANGEL and ESTES (1982) obtained for the field, where they defined straight lines for the log of the power versus degree of harmonic for information between degrees 2 and 12, and between degrees 16 and 23 . The following equations compare the power spectra for the field with that for the field gradient.

$$
\begin{array}{ll}
R_{n}=1.349 \times 10^{9} \times(0.27)^{n} \mathrm{nT}^{2} & 2 \leq n \leq 12, \\
S_{n}=2.529 \times 10^{-4} \times(0.34)^{n}(\mathrm{nT} / \mathrm{m})^{2} & 2 \leq n \leq 12, \\
R_{n}=37.1 \times(0.974)^{n} \mathrm{nT}^{2} & 16 \leq n \leq 23, \\
S_{n}=2.159 \times 10^{-11} \times(1.095)^{n}(\mathrm{nT} / \mathrm{m})^{2} & 16 \leq n \leq 23 .
\end{array}
$$

In these equations, $S_{n}$ is the power of the vertical gradient of the vertical field in each 


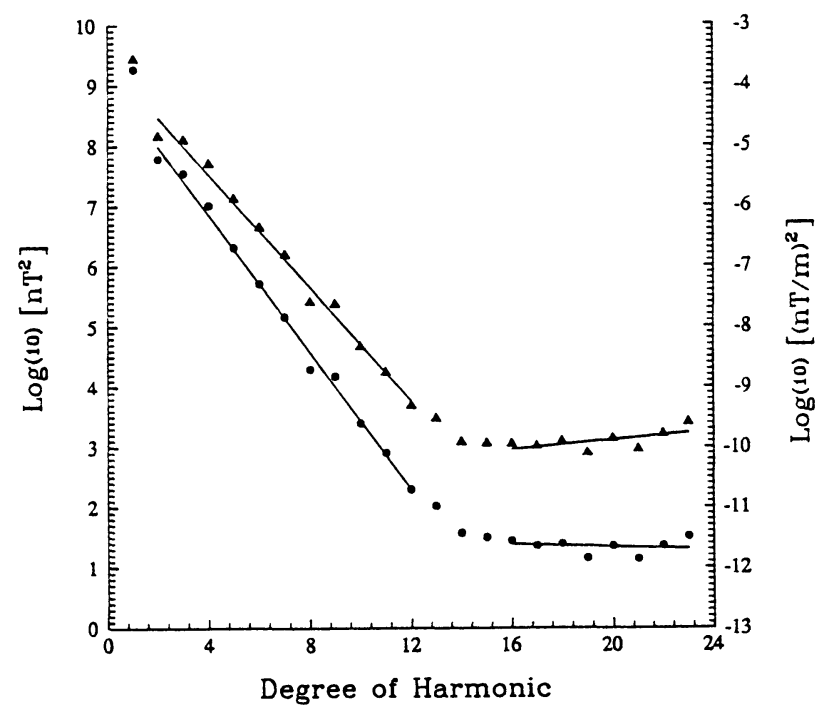

Fig. 1. Mean square field (dots, left hand scale) and vertical gradient of the vertical field (triangles, right hand scale) for the first 23 spherical harmonics of the Earth's field. Data from LANGEL and EsTES (1982). The straight lines plotted in this figure are equations 13 to 16.

degree of harmonic. For the equations describing the behavior at higher degrees of harmonic, the effect of taking the gradient is to change the slope in Fig. 1 from being slightly negative to slightly positive. Although we cannot use these equations to predict what will happen at higher degrees of harmonic than 23 , there are other methods which we can use to suggest that at the wavelength of typical oceanic magnetic anomalies $(10-1000 \mathrm{~km})$ the crustal gradient signal can be almost as strong as the core gradient signal. This will be discussed in greater detail below.

CAIN et al. (1989) have recently produced a model for the spherical harmonic components of the geomagnetic field up to degree 63 , but consider that beyond about degree 48 to 56 the coefficients represent noise. They did not give individual values of the coefficients, but produced equations similar to Eqs. (13) and (15). Their equation for the power in the field as a function of degree of harmonic, at the Earth's surface is as follows.

$$
R_{n}=9.66 \times 10^{8} \times(0.286)^{n}+19.1 \times(0.996)^{n}
$$

This leaves out the noise term. The first part of the equation represents core field, and the second part of the equation represents crustal field. At degree $n$ of 16 the crustal field is much larger than the core field. We can use the expression for the crustal field to determine the total crustal component between degrees 16 and 50. However, this will certainly not capture most of the crustal field at the Earth's surface, since the wavelength cutoff is $800 \mathrm{~km}$. However, the ratios between crustal component between degrees 16 and 50 is compared with the core component between degrees 1 and 12 in the last line of Table 1. These results also emphasize the increasing value of the crustal component of the gradient compared with the core component. 
Table 1 .

\begin{tabular}{lccc}
\hline \multicolumn{4}{c}{ Root mean square components } \\
\hline Component & $\begin{array}{c}\text { Total field } \\
\text { (nT) }\end{array}$ & $\begin{array}{c}\text { Vertical field } \\
\text { (nT) }\end{array}$ & $\begin{array}{c}\text { Gradient } \\
(\mathrm{nT} / \mathrm{m})\end{array}$ \\
\hline $\begin{array}{c}\text { Degree 1 } \\
\text { Degrees 1 to 12 }\end{array}$ & 43231 & 35299 & $1.662 \times 10^{-2}$ \\
& 44488 & 36200 & $1.762 \times 10^{-2}$ \\
\hline Ratios of R MS signals & \\
\hline Deg 13 & $2.30 \times 10^{-4}$ & $2.04 \times 10^{-4}$ & $9.85 \times 10^{-4}$ \\
$\frac{\text { Degs 1 to 12 }}{\text { Degs 16 to 50 }}$ & $5.44 \times 10^{-4}$ & $4.77 \times 10^{-4}$ & $5.53 \times 10^{-3}$ \\
\hline Degs 1 to 12 & & & \\
\hline
\end{tabular}

It is often assumed that spherical harmonics from degree 1 to 12 represent the core field, but that at higher degrees the core field is masked by the crustal field. Therefore it is useful to know the total strength of the core field and other field components, which are shown in Table 1. The third line shows the ratio of the RMS degree 13 quantity divided by the RMS of the sum of the first twelve harmonics, and it can be seen that for the gradient, the degree 13 value is relatively about five times as important as it is in the field.

\section{Sea Floor Spreading Anomalies}

These are lineated magnetic anomalies produced by the interaction of two phenomena. One is sea floor spreading, which produces new oceanic crust at the ridge crests such that crust of equal age is distributed in strips running sub-parallel to the ridge crest. For symmetric spreading, the strips are equally spaced on either side of the ridge crest. The rate at which this occurs varies between 10 and $100 \mathrm{~km} /$ my. Sometimes the lineated pattern is offset by fracture zones, which result from transform faults offsetting the actively spreading ridge crests.

The second phenomenon is that the Earth's magnetic field reverses itself in a random fashion. For well over half of the total area of the oceanic crust, formed during the past $75 \mathrm{my}$, the rate of reversal of the Earth's magnetic field has changed slowly, from giving an average polarity interval of $0.5 \mathrm{my}$ at age $70 \mathrm{my}$ to an average polarity interval of 0.2 my at age $5 \mathrm{my}$. If the straight line graph of average polarity interval length as a function of age is continued to zero age, the average polarity interval would be 0.1 my (HARRISON, 1969; MCFADDEN and MERRILL, 1984).

It is usually believed that the process causing reversals is random, resulting in reversals occurring with a Poisson distribution, or the intervals of constant polarity occurring as an exponential distribution (COX, 1968; HARRISON, 1969; MCFADDEN, 1984). Actual observations of reversals tend to show a gamma distribution (MCFADDEN, 1984) because some of the shorter intervals are not observed in the record. A reversal pattern occurring as a Poisson distribution has a well known power spectrum, and so it is possible to derive the power spectrum for the field resulting from such a magnetization distribution provided that the correct geological information is used in calculating the forward model. 
The power spectrum for a Poisson distribution is given by

$$
P(k)=\frac{2 \mu s A^{2}}{\pi\left(\mu^{2}+\left(k^{2} s^{2} / 4\right)\right)}
$$

where $\mu$ is the rate of reversals per million years, $k$ is the wavenumber in radians $/ \mathrm{km}, s$ is the spreading rate in $\mathrm{km} /$ my and $A$ is the amplitude of the magnetization change in $\mathrm{A} / \mathrm{m}$. Multiplication of this function by the square of the Earth filter (SCHOUTEN and MCCAMY, 1972) will produce the power spectrum of the magnetic field. The Earth filter is given by

$$
f(k)=2 \pi \exp (-k h) \times(1-\exp (-k d))
$$

where $h$ is the depth of the top of the magnetized layer and $d$ is the thickness of the magnetized layer in $\mathrm{km}$.

In order to determine the power spectrum of a field gradient it is necessary to differentiate by a spatial dimension, and in the case of a lineated magnetic anomaly, the spatial dimension may either be vertical or horizontal and perpendicular to the lineation. Differentiation by a spatial dimension is equivalent to multiplication by the wavenumber, and since the power is the square of the amplitude, the power spectrum of one of these gradients is equal to the power spectrum of the field multiplied by $k^{2}$.

This is shown as follows. We assume a magnetization lineated along the $y$ direction, and a magnetization in the $z$ direction. The magnetization is expressed as a Fourier series, one component being given by

$$
M_{z}=M(k) \times \sin (k x) .
$$

In this case the fields in the $x$ and $z$ directions are given by

$$
\begin{aligned}
& H_{z}=f(k) \times M(k) \times \sin (k x), \\
& H_{x}=f(k) \times M(k) \times \cos (k x) .
\end{aligned}
$$

The gradients for this Fourier component are

$$
\begin{aligned}
& \frac{\partial H_{z}}{\partial z}=k H_{z}, \\
& \frac{\partial H_{x}}{\partial x}=-k H_{z}, \\
& \frac{\partial H_{z}}{\partial x}=k H_{x}, \\
& \frac{\partial H_{x}}{\partial z}=k H_{x} .
\end{aligned}
$$

All of the other elements of the tensor have no contribution from this crustal source. 
In general however, all components will have some contribution from crustal sources because the profile will not cross the lineations in a perpendicular direction. However, by rotating the tensor in the manner outlined in Eqs. (1)-(4) the variation in some components can be minimized, thus determining the lineation direction. A similar exercise can be done for horizontal components of magnetization, with similar results.

One example of the comparison between a power spectrum for the field and the field gradient is shown in Fig. 2. This is for a top depth of $5 \mathrm{~km}$, a thickness of $5 \mathrm{~km}$ and the ratio between spreading rate and reversal rate of $8 \mathrm{~km}$ (i.e. a spreading rate of $32 \mathrm{~km} / \mathrm{my}$ and a reversal rate of 4 per my will give this quantity). Figure 2 shows that the maximum in the gradient has been shifted towards shorter wavelengths and occurs at a wavelength of $29 \mathrm{~km}$, compared to a wavelength of $57 \mathrm{~km}$ for the field.

The position of the wavenumber of the maximum in the Earth filter for gradients can be quantified as a function of depth and thickness. This is shown in Fig. 3. For comparison, Fig. 5 in HARRISON (1987a) shows the wavenumber for the position of the maximum in the Earth filter for the field. These two figures show that the position of the maximum occurs at about twice the wavenumber (half the wavelength) for gradients compared with fields. SPECTOR and GRANT (1970) have shown that in the case of unlineated anomalies, the 2-dimensional power spectrum also has a maximum which depends on the depth and the thickness. If one were to perform a 2-dimensional survey with the hope of seeing the maximum, the area covered and hence the effort necessary would only be one quarter for the gradient compared with the field.

An important quantity is the signal carried by the lineated features compared with the signal carried by the core sources. Table 2 shows values for the RMS field generated between wavenumbers of 0.02 and 2 per $\mathrm{km}$ for a number of different models, in which the ratio between spreading rate and reversal rate has been changed from 1 to $64 \mathrm{~km}$. It can be seen by comparison with Table 1 that the crustal gradient signal is comparable in size to the core signal, whereas for the field, the crustal signal is a factor of 300 less than the core signal, which emphasizes one of the uses of gradient measurements, namely that the core signal is relatively much less important. This also indicates that the criterion for orientation in order to extract a crustal signal is much less severe. We have seen that in order to measure the crustal signal to $1 \mathrm{nT}$ (about $1 / 150$ of its signal) we would need an orientation to $1 / 50000$ radians. In order to measure the gradient to $1 / 150$ of its signal we would only need to orient to $1 / 300$ radians.

Another important aspect of studying lineated magnetic anomalies is to learn something about the source of the anomalies. One of the simplest things which would be useful to know is the depth extent of the magnetic source within the oceanic crust. Some models call for a layer only $0.5 \mathrm{~km}$ thick (TALWANI et al., 1971), being essentially confined to the pillow lava layer of the oceanic crust, which can roughly be correlated with seismic layer 2A. Other models (HARRISON, 1987b) call for a much thicker layer, possibly consisting of the whole of the oceanic crust, which is about $5 \mathrm{~km}$ thick in most places. The question is whether the magnetic anomalies can be used to distinguish between the two end member models. One important characteristic is that the simple minded models of completely lineated crust call for a maximum in the power spectrum (HARRISON, 1976) which is in fact rarely if ever seen, possibly due to non-lineated sources (SHURE and PARKER, 1981). However, for the gradient measurements, it is probable that a maximum will be seen for a variety of reasons. Firstly, as has been mentioned above, the core field gradient is relatively much less important than the core 


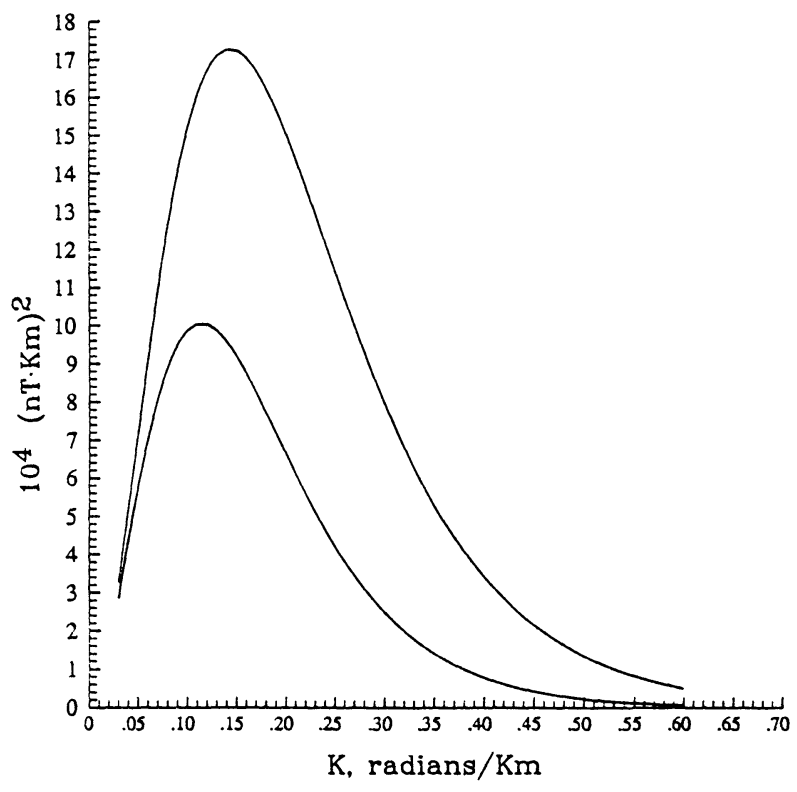

(a)

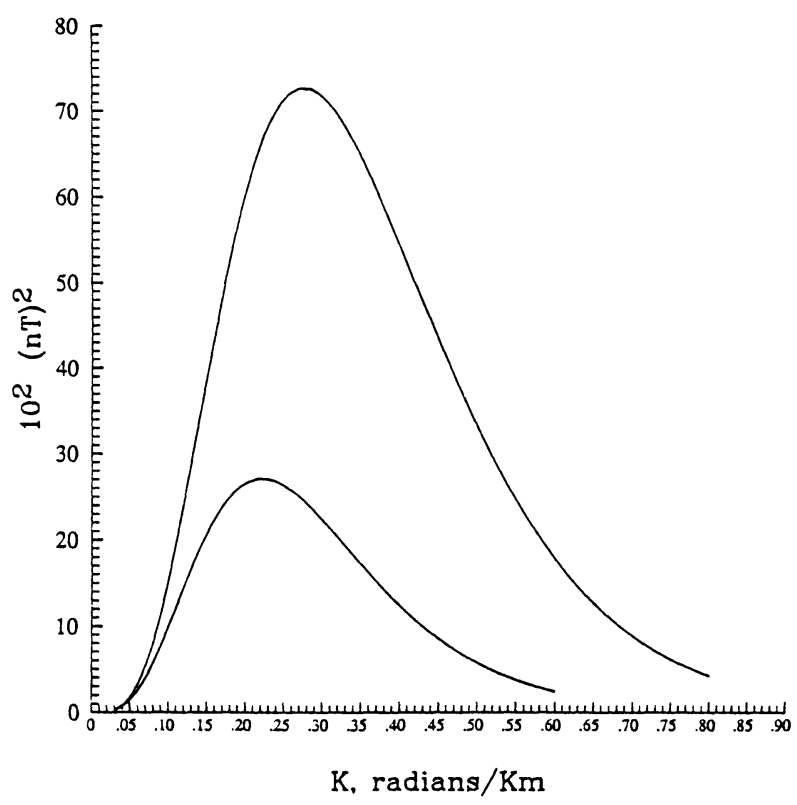

(b)

Fig. 2. Power spectrum of the crustal field (a) and a component of the field gradient (b) for two models of sea floor spreading anomalies. The larger of the two curves in each case is for a crustal thickness of $0.5 \mathrm{~km}$, and the smaller for a crustal thickness of $5.0 \mathrm{~km}$. The magnetization is $1 \mathrm{~A} / \mathrm{m}$ for the $5 \mathrm{~km}$ thick model and 10 $\mathrm{A} / \mathrm{m}$ for the $0.5 \mathrm{~km}$ thick model. 


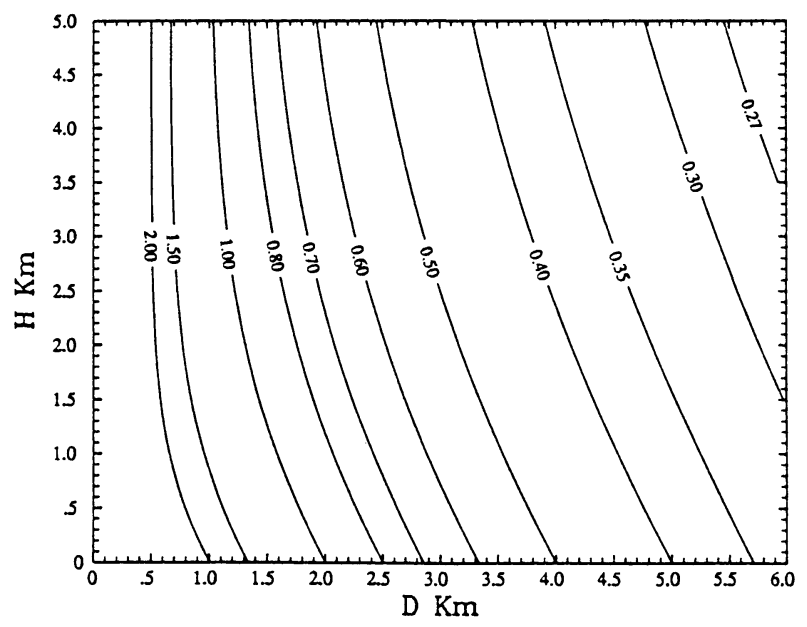

Fig. 3. Value of the wavenumber for the position of the maximum of the Earth filter for gradients as a function of depth to the top of the layer $(h)$ and thickness of the layer $(d)$, both expressed in $\mathrm{km}$. The wavenumber is in radians $/ \mathrm{km}$.

Table 2. Root mean square fields and gradients for crustal sources.

\begin{tabular}{ccc}
\hline $\begin{array}{c}\text { Spreading rate/ } \\
\text { Reversal rate }\end{array}$ & $\begin{array}{c}\text { RMS field } \\
\text { (nT) }\end{array}$ & $\begin{array}{c}\text { RMS field gradient } \\
\text { (nT/km) }\end{array}$ \\
\hline 1 & 64 & 16 \\
2 & 89 & 22 \\
4 & 118 & 27 \\
8 & 143 & 28 \\
16 & 154 & 26 \\
32 & 146 & 21 \\
64 & 123 & 16 \\
\hline
\end{tabular}

field, and so will not produce such a masking of the crustal data. Secondly, the maximum in power spectrum for the gradient is at larger wavenumbers than that for the field, and differences in models of different magnetized layer thickness are greater. Figure 2 shows power spectra for the field (top graphs) and for the field gradient (bottom graphs) for models where the depth to the body is $5 \mathrm{~km}$ and the thickness is either $5 \mathrm{~km}$ or $0.5 \mathrm{~km}$. In order to produce spectra of approximately the same amplitude, the magnetization for the $0.5 \mathrm{~km}$ model is $10 \mathrm{~A} / \mathrm{m}$, ten times as large as for the $5 \mathrm{~km}$ thick model, thus equalizing the total magnetic moment of material per unit area in each model.

Other calculations have been done for a variety of models in which the ratio between spreading rate and reversal rate has been varied between 1 and $64 \mathrm{~km}$, in order to determine the position of the maximum of the power spectrum. These results are shown in Table 3, where it can be seen that the difference between the maxima for models with a $0.5 \mathrm{~km}$ thick magnetized layer compared with a $5.0 \mathrm{~km}$ thick layer are considerably larger 
Table 3. Positions of maxima in spectra, wavenumber (radians $/ \mathrm{km}$ ).

\begin{tabular}{cccccc}
\hline$s / \mu, \mathrm{km}$ & \multicolumn{2}{c}{ Field model thickness } & & \multicolumn{2}{c}{ Gradient model thickness } \\
\cline { 2 - 3 } \cline { 5 - 6 } & $0.5 \mathrm{~km}$ & $5.0 \mathrm{~km}$ & & $0.5 \mathrm{~km}$ & $5.0 \mathrm{~km}$ \\
\hline 1 & 0.19 & 0.14 & & 0.38 & 0.29 \\
2 & 0.18 & 0.14 & & 0.36 & 0.28 \\
4 & 0.17 & 0.13 & & 0.32 & 0.26 \\
8 & 0.14 & 0.11 & & 0.28 & 0.22 \\
16 & 0.11 & 0.09 & & 0.23 & 0.18 \\
32 & 0.08 & 0.07 & & 0.21 & 0.16 \\
64 & 0.05 & 0.04 & & 0.20 & 0.15 \\
\hline
\end{tabular}

for the gradient than for the field. It therefore appears possible to refine our understanding of the bodies responsible for the Vine-Matthews lineations using gradients.

The field has also been used to distinguish a variety of crustal models more complex than the simple uniformly magnetized models of varying thickness discussed above. Some of these are described by CANN (1974), CANDE (1976), CANDE and KENT (1976), KIDD (1977) and BLAKELY and LYNN (1977). Since the distinction between the more complex and simpler models depends on subtle differences on the magnetic anomaly, it is to be expected that gradient measurements would help to distinguish between the various models which have been proposed.

\section{Time Varying Components of the Gradient}

One problem which has to be faced when measuring magnetic fields is that the external component of the field can cause a significant error in the measurement of the internal field. External effects can be corrected by recording the field at one spot during the time of a survey, but although this is usually done when the observations are to be used for commercial purposes, it is not common when the objective is scientific research. Although HARRISON and CARLE (1981) showed that the normal diurnal variation of the field does not add much to the power spectrum measured over a very long magnetic profile in the Pacific Ocean, we shall now demonstrate that normal diurnal variations are much less important in field gradient measurements than in field measurements.

We assume that the diurnal variation has an amplitude of $50 \mathrm{nT}$ and is assumed to have a signal representing one half of a sine wave lasting for the daylight hours. Therefore its wavelength is equivalent to the circumference of the Earth. Thus the gradient of the variation has an amplitude of $7.9 \times 10^{-6} \mathrm{nT} / \mathrm{m}$. Comparison of these two numbers with the relevant crustal signals shown in Table 2 indicates how much less important the diurnal variation is in gradient measurements than in field measurements. Both the field and the field gradient will appear as spatially varying signals of the above amplitudes to observers who are making surveys at speeds small compared with the Earth's rotation rate. HARRISON et al. (1975) have described magnetic observations over a number of seamounts, and typically the seamount may have a maximum anomaly whose strength is $500 \mathrm{nT}$. A simple calculation based on some of the seamounts discussed in the above paper indicates that the maximum gradient which might be measured over such a seamount is of the order of $50 \mathrm{nT} / \mathrm{km}$. Thus the signal to noise ratio for the field 
measurements is approximately 10, whereas that for the gradient measurements it is about 5000 .

Magnetic storms can also be shown to have a relatively smaller effect on gradients than on fields. Occasionally these can produce anomalies at the surface of the Earth of $1000 \mathrm{nT}$, i.e. as large as or larger than typical crustal anomalies. These fields are caused by electric currents flowing in the magnetosphere and ionosphere, but if we assume that they are caused by dipole-like sources (which will give the largest gradient-to-field ratio, compared to any distributed source) at an elevation of $150 \mathrm{~km}$, then the maximum gradient will be $20 \times 10^{-3} \mathrm{nT} / \mathrm{m}$, relatively much smaller than the field, as can be seen in Table 2 .

\section{Proposed Instrument}

In order to make measurements of field gradients, a superconducting quantum interference device (SQUID) magnetometer is used. In a gradient mode this instrument has many advantages over other instruments (FALCO and SCHULLER, 1981). Compared with the normal geophysical use of magnetization measurements for paleo- or rockmagnetism, the Squid magnetometer used for gradient measurements has its coils reversed so that they measure the difference in flux linkage. It is simple to show that the sensitivity depends on the area of the coils times their separation, in other words on the volume enclosed by the coils. For normal applications a Squid gradiometer can be made with a sensitivity of $2 \times 10^{-4} \mathrm{nT} / \mathrm{m}$ (GILLESPIE et al., 1972), a factor of 80 smaller than the signals given in Table 2 . Increasing the linear dimension should allow sensitivities of $5 \times 10^{-6} \mathrm{nT} / \mathrm{m}$ to be achieved easily (HASTINGS et al., 1985).

It has also been proposed to use magnetic gradiometers for satellite applications (HASTINGS et al., 1985). In this case, the sensitivity needs to be increased because of the distance to the crustal sources. HASTINGS et al. (1985) have shown that a sensitivity of $10^{-6} \mathrm{nT} / \mathrm{m}$ is necessary to measure these crustal gradients accurately. For this, the Squid linear dimension has to be about $0.5 \mathrm{~m}$. HASTINGS et al. (1985) have also done preliminary calculations pointing out the advantages of space gradiometer measurements. They show that at a nominal altitude of $600 \mathrm{~km}$, the value of $G_{\text {crust }} / G_{\text {external }}$ is a factor of ten larger than $B_{\text {crust }} / B_{\text {external, }}$, and that $G_{\text {crust }} / G_{\text {core }}$ is a factor of three larger than $B_{\text {crust }} / B_{\text {core }}$, where $G$ is a gradient and $B$ is a field.

This indicates that gradient measurements are relatively better for studying crustal anomalies, even at satellite altitudes. But surprisingly, HASTINGS et al. (1985) also show that at satellite altitudes $G_{\text {core }} / G_{\text {external }}$ is greater than $B_{\text {core }} / B_{\text {external }}$, indicating that there are also some advantages in looking at core sources by measuring gradients from space. However $G_{\text {core }} / G_{\text {crust }}$ is less than $B_{\text {core }} / B_{\text {crust }}$. These comparisons need to be repeated, as HASTINGS et al. (1985) used a very simple model for the external field.

Oddly, there was little discussion of Squid uses in studies of the Earth's magnetic field in the conference held on Squid Applications to Geophysics (WEINSTOCK and OVERTON, 1981), although Squids have been used extensively in paleomagnetic research for the past two decades.

6. Rotationally Invariant Scalars

Even if no orientation is available, some information may be obtained by measure- 
ment of the gradient tensor. This is because there are some rotationally invariant scalars which can be calculated. If field components are also available, the number of rotationally invariant scalars increases.

The simplest is the total field intensity, which can be measured directly, as is done with a proton precession magnetometer or optically pumped magnetometers such as cesium and rubidium vapor magnetometers. The total field intensity can also be measured by determining the three orthogonal components of the field.

$$
R_{1}=\left(H_{x}^{2}+H_{y}^{2}+H_{z}^{2}\right)^{1 / 2}
$$

This method was used to good effect in the Magsat satellite, where the total field instrument (a cesium vapor magnetometer) did not work satisfactorily, and where the orientation of the vector instrument (a three-component fluxgate magnetometer) was sometimes suspect. But the total field amplitude could be calculated from the component data.

The next most useful rotationally invariant scalar is the trace of the product matrix of the tensor times itself.

$$
R_{2}=\operatorname{Tr}\left(T_{i j} \cdot T_{j i}\right)
$$

Suppose that there are structures lineated in the $y$ direction, then in the absence of a regional field or regional field gradient this second rotationally invariant scalar reduces to the following.

$$
R_{2}=\left(T_{x x}\right)^{2}+\left(T_{z z}\right)^{2}+2\left(T_{x z}\right)^{2}
$$

therefore

$$
R_{2}=2[k M(k) f(k)]^{2}
$$

This shows that there will be no horizontal spatial dependence to the signal for this second rotationally invariant scalar in the absence of a regional field gradient. A similar thing would happen if the total field amplitude were to be measured over lineated features in the absence of a regional field. In this case, the amplitude (calculated from Eqs. (19) and (20)) would be given by $f(k) M(k)$.

However in the presence of regional field gradients, it is possible to show that the second rotationally invariant scalar would have the form

$$
R_{2}=C_{x x} \times T_{x x}+C_{x z} \times T_{x z}+C_{r}+2 \times[k \times M(k) \times f(k)]^{2}
$$

where $C_{x x}$ and $C_{x z}$ are sums of regional gradients and $C_{r}$ is the sum of products of regional gradients. Thus the signal represents a coupling of the regional field gradient and the gradient produced by the lineated structure. It should be possible to uncouple these two signatures by employing a spherical harmonic model for the regional field gradient, in much the same way as regional fields are removed from total field signals using spherical harmonics.

Other potentially useful rotationally invariant scalars are as follows. 


$$
\begin{aligned}
& R_{3}=H_{i} T_{i j} H_{j}, \\
& R_{4}=\left(T_{i j} H_{j}\right) \cdot\left(T_{i k} H_{k}\right), \\
& R_{5}=\operatorname{Tr}\left(T_{i j} T_{j k} T_{k i}\right) .
\end{aligned}
$$

These higher order rotational invariants introduce modulation of the magnetization by a function of fractional wavenumbers. This modulation enhances the signal of invariants at small wavenumbers relative to the actual magnetization signature. The decoupling of the regional and crustal signals is more complicated for the higher order rotational invariants.

\section{Conclusions}

We have discussed magnetic field gradients in terms of their usefulness in modelling crustal magnetizations. It is shown that gradients enhance shorter wavelength features compared to field measurements. This is an advantage in that both the core signal and the signal from external fields, both of which appear as long wavelength signals, are reduced compared with the crustal signal. Measurements of gradients will therefore allow better models of crustal magnetization to be calculated. If the gradient device can be oriented, then directions of lineation can be determined from single profiles, and anomalies caused by unlineated sources can be identified. The accuracy of orientation is not nearly as severe as it is for measuring field components if crustal signatures are of interest.

Research supported by the National Aeronautics and Space Administration, Grant NAG-5414. Contribution from the University of Miami, Rosenstiel School of Marine and Atmospheric Science.

\section{REFERENCES}

BLAKELY, R. J. and A. Cox, Identification of short polarity events by transforming marine magnetic profiles to the pole, J. Geophys. Res., 77, 4339-4349, 1972.

Blakely, R. J. and W. S. LynN, Reversal transition widths and fast-spreading centers, Earth Planet. Sci. Lett., 33, 321-330, 1977.

Blakely, R. J., A. Cox, and E. J. IUfER, Vector magnetic data for detecting short polarity intervals in marine magnetic profiles, J. Geophys. Res., 78, 6977-6983, 1973.

Cain, J. C., Z. WANG, D. R. SchmitZ, and J. MEYer, The geomagnetic spectrum for 1980 and core-crustal separation, Geophys. J., 97, 443-447, 1989.

CAnde, S. C., A palaeomagnetic pole from Late Cretaceous marine magnetic anomalies in the Pacific, Geophys. J. R. Astron. Soc., 44, 547-566, 1976.

CANDE, S. C. and D. V. KENT, Constraints imposed by the shape of marine magnetic anomalies on the magnetic source, J. Geophys. Res., 81, 4157-4162, 1976.

Cann, J. R., A model for oceanic crustal structure developed, Geophys. J. R. Astron. Soc., 39, 169-187, 1974.

Cox, A., Lengths of geomagnetic polarity intervals, J. Geophys. Res., 73, 3247-3260, 1968.

FAlco, C. M. and I. K. Schuller, Squids and their sensitivity for geophysical applications, in Squid Applications to Geophysics, edited by H. Weinstock and W. C. Overton, Jr., 208 pp., Soc. Explor. Geophys., Tulsa, Oklahoma, 1981.

Gillespie, G. H., W. N. Podney, and J. L. Buxton, Low-frequency noise spectra of a superconductivity magnetic gradiometer, J. Appl. Phys., 48, 354-357, 1972.

Harrison, C. G. A., What is the true rate of reversals of the Earth's magnetic field?, Earth Planet. Sci. Lett., 6, 186-188, 1969. 
Harrison, C. G. A., Magnetization of the oceanic crust, Geophys. J. R. Astron. Soc., 47, 257-284, 1976.

Harrison, C. G. A., The crustal field, in Geomagnetism, Vol. 1, edited by J. A. Jacobs, pp. 513-610, Academic Press, London, 1987a.

Harrison, C. G. A., Marine magnetic anomalies-the origin of the stripes, Ann. Rev. Earth Planet. Sci., 15, 503-543, 1987b.

Harrison, C. G. A. and H. M. CARLE, Intermediate wavelength magnetic anomalies over ocean basins, $J$. Geophys. Res., 86, 11,585-11,599, 1981.

Harrison, C. G. A., R. Jarrard, V. Vacquier, and R. L. Larson, Palaeomagnetism of Cretaceous Pacific seamounts, Geophys. J. R. Astron. Soc., 42, 859-882, 1975.

Hastings, R., R. P. S. Mahler, R. Schneider, Jr., and J. H. Eraker, Cryogenic magnetic gradiometers for space applications, IEEE Trans. Geosci. Remote Sensing, GE-23, 552-561, 1985.

Hood, P., Gradient measurements in aeromagnetic surveying, Geophysics, 30, 891-902, 1965.

Hood, P. A., A new mapping tool for mineral exploration, The Northern Miner, 61, pp. A1, A20-21, Nov. 27, 1975.

HooD, P., Aeromagnetic gradiometry: a superior geological mapping tool for mineral exploration programs, in Squid Applications to Geophysics, edited by H. Weinstock and W. C. Overton, Jr., pp. 72-77, Soc. Explor. Geophys., 1981.

Hood, P. and D. J. McClure, Gradient measurements in ground magnetic prospecting, Geophysics, 30, 403-410, 1965.

Hood, P. J., M. T. Holroyd, and P. H. McGrath, Magnetic methods applied to base metal exploration, in Geophysics and Geochemistry in the Search for Metallic Ores, edited by P. J. Hood, Geol. Surv. Canada Economic Geology Report 31, pp. 77-104, 1979.

ISEZAKI, N., A new shipboard three-component magnetometer, Geophysics, 51, 1992-1998, 1986.

KIDD, R. G. W., The nature and shape of the sources of marine magnetic anomalies, Earth Planet. Sci. Lett., 33, 310-320, 1977.

LAngel, R. A. and R. H. Estes, A geomagnetic field spectrum, Geophys. Res. Lett., 9, 250-253, 1982.

LowEs, F. J., Mean-square values on sphere of spherical harmonic vector fields, J. Geophys. Res., 71, 2179, 1966.

Mason, R. G., A magnetic survey off the west coast of the United States between latitudes $32^{\circ}$ and $36^{\circ} \mathrm{N}$, longitudes $121^{\circ}$ and $128^{\circ}$ west, Geophys. J. R. Astron. Soc., 1, 320-329, 1958.

Mason, R. G. and A. D. RAFF, Magnetic survey off the west coast of North America, $32^{\circ} \mathrm{N}$ latitude to $42^{\circ} \mathrm{N}$ latitude, Geol. Soc. Amer. Bull., 72, 1259-1266, 1961.

Mauersberger, P., Das mittel der energie-dichte des geomagnetischen hauptfeldes an der erdoberfläche und seine säkulare, Abderung, Gerlands Beitr. Geophys., 65, 207-215, 1956.

McFadden, P. L., Statistical tools for the analysis of geomagnetic reversal sequences, J. Geophys. Res., 89, 3363-3372, 1984.

McFadden, P. L. and R. T. Merrill, Lower mantle convection and geomagnetism, J. Geophys. Res., 89, 3354-3362, 1984.

Schouten, H. and K. MCCAMy, Filtering marine magnetic anomalies, J. Geophys. Res., 77, 7089-7099, 1972.

SeAma, N. and N. IsEZAKI, Sea-floor magnetization in the eastern part of the Japan Basin, Tectonophysics, 181, 285-297, 1990.

Shure, L. and R. L. Parker, An alternative explanation for intermediate-wavelength magnetic anomalies, $J$. Geophys. Res., 86, 11,600-11,608, 1981.

SPECTOR, A. and F. S. GRANT, Statistical models for interpreting aeromagnetic data, Geophysics, 35, 293-302, 1970.

Talwani, M., C. C. Windisch, and M. G. LAngSeth, Jr., Reykjanes ridge crest: a detailed geophysical study, J. Geophys. Res., 76, 473-517, 1971.

Vestine, E. H. and N. Davids, Analysis and interpretation of geomagnetic anomalies, Terr. Magn. Atmos. Elect., 50, 1-36, 1945.

Weinstock, H. and W. C. Overton, Jr., Squid Applications to Geophysics, 208 pp., Society of Exploration Geophysicists, Tulsa, Oklahoma, 1981. 